\title{
THE BOGOLIUBOV WEAKLY IMPERFECT BOSE-GAS
}

\author{
Valentin A. Zagrebnov \\ Centre de Physique Théorique (Unité Propre de Recherche 7061), \\ Université de la Méditerranée (Aix-Marseille II) \\ CNRS - Luminy - Case 907, 13288 Marseille, Cedex 09, France
}

(Received July 23, 1999)

\begin{abstract}
It is shown that the condensation in the Bogoliubov Weakly Imperfect Bose-Gas (WIBG) may appear in two stages. If the interaction is such that the pressure of the WIBG does not coincide with the pressure of the Perfect Bose-Gas (PBG), then the WIBG may manifest two kinds of condensations: a non-conventional condensation in the zero-mode due to the interaction (the first stage) and a conventional (generalized of type I) Bose-Einstein condensation in modes next to the zero-mode due to the particle density saturation (the second stage).

Key words: Bogoliubov Weakly Imperfect Gas, non-conventional Bose-condensation, generalized (type I) condensation.
\end{abstract}

PACS numbers: 03.75.Fi, 05.30.Jp

\section{INTRODUCTION}

Consider a system of bosons of mass $m$ in a cubic box $\Lambda=L \times L \times L \subset \mathbb{R}^{3}$ of the volume $V \equiv|\Lambda|=L^{3}$, with periodic boundary conditions on $\partial \Lambda$. If $\varphi(x)$ denotes an absolutely integrable two-body interaction potential and

$$
v(q)=\int_{\mathbb{R}^{3}} d^{3} x \varphi(x) e^{-i q x}, q \in \mathbb{R}^{3},
$$

then its second-quantized Hamiltonian acting on the boson Fock space $\mathcal{F}_{\Lambda}$ can be written as

$$
\begin{aligned}
H_{\Lambda} & =\sum_{k \in \Lambda^{*}} \varepsilon_{k} a_{k}^{*} a_{k} \\
& +\frac{1}{2 V} \sum_{k_{1}, k_{2}, q \in \Lambda^{*}} v(q) a_{k_{1}+q}^{*} a_{k_{2}-q}^{*} a_{k_{1}} a_{k_{2}}
\end{aligned}
$$

where all sums run over the set $\Lambda^{*}$ defined by

$$
\begin{aligned}
\Lambda^{*}=\left\{k \in \mathbb{R}^{3}: \alpha\right. & =1,2,3, \\
k_{\alpha} & \left.=\frac{2 \pi n_{\alpha}}{L} \text { et } n_{\alpha}=0, \pm 1, \pm 2, \ldots\right\} .
\end{aligned}
$$

Here $\varepsilon_{k}=\hbar^{2} k^{2} / 2 m$ is the kinetic energy, and $a_{k}^{\#}=$ $\left\{a_{k}^{*}, a_{k}\right\}$ are usual boson creation and annihilation operators in the one-particle state $\psi_{k}(x)=V^{-\frac{1}{2}} e^{i k x}, k \in \Lambda^{*}$, $x \in \Lambda$; for example, $a_{k}^{*} \equiv a^{*}\left(\psi_{k}\right)=\int_{\Lambda} d x \psi_{k}(x) a^{*}(x)$ where $a^{\#}(x)$ are basic boson operators in the Fock space $\mathcal{F}_{\Lambda}$ over $L^{2}(\Lambda)$

Below we suppose that:

(A) $\varphi(x)=\varphi(\|x\|)$ and $\varphi \in L^{1}\left(\mathbb{R}^{3}\right)$

(B) $v(k)$ is a real continuous function, satisfying $v(0)>0$ and $0 \leq v(k) \leq v(0)$ for $k \in \mathbb{R}^{3}$.

If one expects that Bose-Einstein condensation, which occurs for the Perfect Bose-Gas (PBG) in the mode $k=0$, persists for a weak interaction $\varphi(x)$, then according to Bogoliubov [1,2] the most important terms in (1.2) should be those in which at least two operators $a_{0}^{*}, a_{0}$ appear. We are thus led to consider the following truncated Hamiltonian (the Bogoliubov Hamiltonian for a Weakly Imperfect Bose gas (WIBG), see $[1,2]$ ):

$$
H_{\Lambda}^{B}=T_{\Lambda}+U_{\Lambda}^{D}+U_{\Lambda}
$$

where

$$
\begin{aligned}
T_{\Lambda} & =\sum_{k \in \Lambda^{*}} \varepsilon_{k} a_{k}^{*} a_{k}, \\
U_{\Lambda}^{D} & =\frac{v(0)}{V} a_{0}^{*} a_{0} \sum_{k \in \Lambda^{*}, k \neq 0} a_{k}^{*} a_{k} \\
& +\frac{1}{2 V} \sum_{k \in \Lambda^{*}, k \neq 0} v(k) a_{0}^{*} a_{0}\left(a_{k}^{*} a_{k}+a_{-k}^{*} a_{-k}\right) \\
& +\frac{v(0)}{2 V} a_{0}^{*^{2}} a_{0}^{2}, \\
U_{\Lambda} & =\frac{1}{2 V} \sum_{k \in \Lambda^{*}, k \neq 0} v(k)\left(a_{k}^{*} a_{-k}^{*} a_{0}^{2}+a_{0}^{*^{2}} a_{k} a_{-k}\right) .
\end{aligned}
$$

Notice that the self-adjoint operator $H_{\Lambda}^{B}$ is defined on a dense domain in the boson Fock space $\mathcal{F}_{\Lambda} \approx \mathcal{F}_{0 \Lambda} \otimes \mathcal{F}_{\Lambda}^{\prime}$ over $L^{2}(\Lambda)$, where $\mathcal{F}_{0 \Lambda}$ and $\mathcal{F}_{\Lambda}^{\prime}$ are the boson Fock spaces constructed out of $\mathcal{H}_{0 \Lambda}$ (the one-dimensional subspace generated by $\left.\psi_{k=0} \in L^{2}(\Lambda)\right)$ and of its orthogonal complement $\mathcal{H}_{0 \Lambda}^{\perp}$ respectively.

For any complex $c \in \mathbb{C}$, we can define in $\mathcal{F}_{0 \Lambda}$ a coherent vector 


$$
\psi_{0 \Lambda}(c)=e^{-V|c|^{2} / 2} \sum_{k=0}^{\infty} \frac{1}{k !}(\sqrt{V} c)^{k}\left(a_{0}^{*}\right)^{k} \Omega_{0}
$$

where $\Omega_{0}$ is the vacuum of $\mathcal{F}_{\Lambda}$ and therefore $a_{0} \psi_{0 \Lambda}(c)=c \sqrt{V} \psi_{0 \Lambda}(c)$. Using this concept of the coherent vectors in $\mathcal{F}_{0 \Lambda}$, Ginibre [3] defines the Bogoliubov approximation to a Hamiltonian $H_{\Lambda}$ in $\mathcal{F}_{\Lambda}$ as follows:

Definition 1 The Bogoliubov approximation $H_{\Lambda}\left(c^{\#}, \mu\right)$ for a Hamiltonian $H_{\Lambda}(\mu) \equiv H_{\Lambda}-\mu N_{\Lambda}$ on $\mathcal{F}_{\Lambda}$ is the operator defined on $\mathcal{F}_{\Lambda}^{\prime}$ by its quadratic form

$$
\left(\psi_{1}^{\prime}, H_{\Lambda}\left(c^{\#}, \mu\right) \psi_{2}^{\prime}\right)_{\mathcal{F}_{\Lambda}^{\prime}} \equiv\left(\psi_{0 \Lambda}(c) \otimes \psi_{1}^{\prime}, H_{\Lambda}(\mu) \psi_{0 \Lambda}(c) \otimes \psi_{2}^{\prime}\right)_{\mathcal{F}_{\Lambda}}
$$

for $\psi_{0 \Lambda}(c) \otimes \psi_{1,2}^{\prime}$ in the form-domain of $H_{\Lambda}(\mu)$, where $c^{\#}=(c, \bar{c})$ and

$$
N_{\Lambda}=\sum_{k \in \Lambda^{*}} N_{k}
$$

is the particle-number operator (here $N_{k} \equiv a_{k}^{*} a_{k}$ is the occupation-number operator for the mode $k$ ) and $\mu$ is the chemical potential.

Therefore, the Bogoliubov approximation in the Bogoliubov Hamiltonian for the WIBG (1.4) gets the form:

$$
\begin{aligned}
H_{\Lambda}^{B}\left(c^{\#}, \mu\right) & =\sum_{k \in \Lambda^{*}, k \neq 0}\left[\varepsilon_{k}-\mu+v(0)|c|^{2}\right] a_{k}^{*} a_{k}+\frac{1}{2} \sum k \in \Lambda^{*}, k \neq 0 v(k)|c|^{2}\left[a_{k}^{*} a_{k}+a_{-k}^{*} a_{-k}\right] \\
& +\frac{1}{2} \sum_{k \in \Lambda^{*}, k \neq 0} v(k)\left[c^{2} a_{k}^{*} a_{-k}^{*}+\bar{c}^{2} a_{k} a_{-k}\right]-\mu|c|^{2} V+\frac{1}{2} v(0)|c|^{4} V
\end{aligned}
$$

Then the Hamiltonian (1.9) can be diagonalized (cf. $[1,2])$. The pressure associated with $H_{\Lambda}^{B}\left(c^{\#}, \mu\right)$ :

$$
\tilde{p}_{\Lambda}^{B}\left(\beta, \mu ; c^{\#}\right) \equiv \frac{1}{\beta V} \ln \operatorname{Tr}_{\mathcal{F}_{\Lambda}^{\prime}} e^{-\beta H_{\Lambda}^{B}\left(c^{\#}, \mu\right)},
$$

(where $\theta=\beta^{-1}$ is the temperature) is well-defined for $\mu \leq v(0)|c|^{2}$ and has the following explicit form:

$$
\begin{aligned}
& \tilde{p}_{\Lambda}^{B}\left(\beta, \mu ; c^{\#}\right)=\xi_{\Lambda}(\beta, \mu ; x)+\eta_{\Lambda}(\mu ; x), \\
& \xi_{\Lambda}(\beta, \mu ; x)=\frac{1}{\beta V} \sum_{k \in \Lambda^{*}, k \neq 0} \ln \left(1-e^{-\beta E_{k}}\right)^{-1}, \\
& \eta_{\Lambda}(\mu ; x)=-\frac{1}{2 V} \sum_{k \in \Lambda^{*}, k \neq 0}\left(E_{k}-f_{k}\right)+\mu x-\frac{1}{2} v(0) x^{2},
\end{aligned}
$$

where $x=|c|^{2} \geq 0$ and

$$
\begin{aligned}
f_{k} & =\varepsilon_{k}-\mu+x[v(0)+v(k)], \\
h_{k} & =x v(k), \\
E_{k} & =\sqrt{f_{k}^{2}-h_{k}^{2}} .
\end{aligned}
$$

Another observation concerns the original Hamiltonian $(1.4)$, see $[4,5]$.

Proposition 1 The pressure $p_{\Lambda}^{B}(\beta, \mu)$ associated with the Bogoliubov Hamiltonian $H_{\Lambda}^{B}$, i.e.

$$
p_{\Lambda}^{B}(\beta, \mu) \equiv p_{\Lambda}\left[H_{\Lambda}^{B}\right] \equiv \frac{1}{\beta V} \ln \operatorname{Tr}_{\mathcal{F}_{\Lambda}} e^{-\beta\left(H_{\Lambda}^{B}-\mu N_{\Lambda}\right)},
$$

is defined only in domain $Q=\{\mu \leq 0\} \times\{\theta \geq 0\}$ and it is equal in the thermodynamic limit to

$$
\begin{aligned}
p^{B}(\beta, \mu) & =\sup _{c \in \mathbb{C}} \widetilde{p}^{B}\left(\beta, \mu ; c^{\#}\right) \\
& \equiv \lim _{\Lambda}\left\{\sup _{c \in \mathbb{C}} \widetilde{p}_{\Lambda}^{B}\left(\beta, \mu ; c^{\#}\right)\right\}
\end{aligned}
$$

Therefore, from the explicit form (1.11) of $\widetilde{p}_{\Lambda}^{B}\left(\beta, \mu ; c^{\#}\right)$ we can deduce (cf. $\left.[4,5]\right)$ the following two corollaries:

Corollary 2. Let $v(k)$ satisfy $(A),(B)$ and

$$
v(0) \geq \frac{1}{2(2 \pi)^{3}} \int_{\mathbb{R}^{3}} d^{3} k \frac{v(k)^{2}}{\varepsilon_{k}} .
$$


Then

$$
\begin{aligned}
p^{B}(\beta, \mu) & =\sup _{c \in \mathbb{C}} \widetilde{p}^{B}\left(\beta, \mu ; c^{\#}\right)=\widetilde{p}^{B}(\beta, \mu ; 0) \\
& =p^{P}(\beta, \mu)
\end{aligned}
$$

where

$$
p^{P}(\beta, \mu) \equiv \lim _{\Lambda} p_{\Lambda}\left[T_{\Lambda}\right]
$$

is the pressure of the Perfect Bose-Gas (PBG).

Corollary 3. Let $v(k)$ satisfy $(A),(B)$ and (C) :

$$
v(0)<\frac{1}{2(2 \pi)^{3}} \int_{\mathbb{R}^{3}} d^{3} k \frac{v(k)^{2}}{\varepsilon_{k}} .
$$

Then there are $\mu_{0}<0$ and $\theta_{0}(\mu)>0$ such that one has

$$
\begin{aligned}
p^{B}(\beta, \mu) & =\sup _{c \in \mathbb{C}} \tilde{p}^{B}\left(\beta, \mu ; c^{\#}\right) \\
& =\widetilde{p}^{B}\left(\beta, \mu ; \hat{c}^{\#}(\beta, \mu) \neq 0\right)>p^{P}(\beta, \mu),
\end{aligned}
$$

for $(\theta, \mu) \in D$ defined by

$$
D=\left\{(\theta, \mu): \mu_{0}<\mu \leq 0,0 \leq \theta<\theta_{0}(\mu)\right\},
$$

and

$$
p^{B}(\beta, \mu)=\sup _{c \in \mathbb{C}} \tilde{p}^{B}\left(\beta, \mu ; c^{\#}\right)=p^{P}(\beta, \mu),
$$

for $(\theta, \mu) \notin \bar{D}$.

Moreover, see $[4,5], D$ is a domain which corresponds to a non-conventional condensation in the mode $k=0$ :

$$
\begin{aligned}
\rho_{0}^{B}(\theta, \mu) & \equiv \lim _{\Lambda}\left\langle\frac{a_{0}^{*} a_{0}}{V}\right\rangle_{H_{\Lambda}^{B}}(\beta, \mu)= \\
& =\left\{\begin{array}{c}
|\hat{c}(\beta, \mu)|^{2}>0,(\theta, \mu) \in D \\
0,(\theta, \mu) \in Q \backslash \bar{D}
\end{array}\right\},
\end{aligned}
$$

where $\widehat{c}(\beta, \mu)$ is defined by (1.18) and

$$
\omega_{\Lambda}^{B}(-) \equiv\langle-\rangle_{H_{\Lambda}^{B}}(\beta, \mu)
$$

represents the grand-canonical Gibbs state for the Hamiltonian $H_{\Lambda}^{B}$. The non-conventional Bosecondensate (1.21) undergoes a jump on the boundary $\partial D$, see $[4,5]$.

However, we have to admit that in $[4,5]$ we study the WIBG only in the grand-canonical ensemble, i.e. by fixing the chemical potential $\mu$. On the other hand, it is well-known that the conventional Bose-Einstein condensation in the PBG is parametrized by the total particle density $\rho$ which should be higher than the saturated for $\mu=0$ particle density $\rho^{P}(\beta, \mu)$ in the grand canonical ensemble: $\rho>\rho_{c}^{P}(\theta) \equiv \rho^{P}\left(\theta^{-1}, \mu=0\right)$. Thus [4,5] do not study the conventional Bose-Einstein condensation in the WIBG.

Notice that using the Griffiths Lemma (see [6,7]) and Proposition 1, one finds for the grand-canonical total particle density in the WIBG:

$$
\begin{aligned}
\rho^{B}(\beta, \mu) & \equiv \lim _{\Lambda} \omega_{\Lambda}^{B}\left(\frac{N_{\Lambda}}{V}\right)=\lim _{\Lambda} \frac{1}{V} \sum_{k \in \Lambda^{*}} \omega_{\Lambda}^{B}\left(N_{k}\right)=\lim _{\Lambda} \partial_{\mu} p_{\Lambda}^{B}(\beta, \mu)=\partial_{\mu} \tilde{p}^{B}(\beta, \mu ; 0) \\
& =\frac{1}{(2 \pi)^{3}} \int_{\mathbb{R}^{3}}\left(e^{\beta\left(\varepsilon_{k}-\mu\right)}-1\right)^{-1} d^{3} k,
\end{aligned}
$$

for $(\theta, \mu<0) \in Q \backslash \bar{D}$ and:

$$
\begin{aligned}
\rho^{B}(\beta, \mu) & =\partial_{\mu} \widetilde{p}^{B}\left(\beta, \mu ; \widehat{c}^{\#}(\beta, \mu) \neq 0\right) \\
& =\left.\frac{1}{(2 \pi)^{3}} \int_{\mathbb{R}^{3}}\left[\frac{f_{k}}{E_{k}}\left(e^{\beta E_{k}}-1\right)^{-1}+\frac{h_{k}^{2}}{2 E_{k}\left(f_{k}+E_{k}\right)}\right] d^{3} k\right|_{c=\widehat{c}(\beta, \mu)}+|\widehat{c}(\beta, \mu)|^{2},
\end{aligned}
$$

for $(\theta, \mu<0) \in D$. Then, from (1.23) and (1.24), we see that the total density $\rho^{B}(\beta, \mu)$ reaches its maximal (critical) value at $\mu=0$, i.e. $\rho_{c}^{B}(\theta) \equiv \rho^{B}(\beta, \mu=0)$ :

(i) for $\theta>\theta_{0}(\mu=0)$

$$
\rho_{c}^{B}(\theta)=\frac{1}{(2 \pi)^{3}} \int_{\mathbb{R}^{3}}\left(e^{\beta \varepsilon_{k}}-1\right)^{-1} d^{3} k<+\infty,
$$


(ii) for $\theta<\theta_{0}(\mu=0)$

$$
\rho_{c}^{B}(\theta)=\left.\frac{1}{(2 \pi)^{3}} \int_{\mathbb{R}^{3}}\left[\frac{f_{k}}{E_{k}}\left(e^{\beta E_{k}}-1\right)^{-1}+\frac{h_{k}^{2}}{2 E_{k}\left(f_{k}+E_{k}\right)}\right] d^{3} k\right|_{\substack{c=\hat{c}(\beta, 0) \\ \mu=0}}+|\widehat{c}(\beta, \mu=0)|^{2}<+\infty .
$$

By convexity of $p^{B}(\beta, \mu)$ with respect to the parameter $\mu$ one gets that

$$
\lim _{\mu \rightarrow \mu_{0}(\theta)^{-}} \rho^{B}(\beta, \mu) \equiv \rho_{\mathrm{inf}}^{B}(\theta)<\lim _{\mu \rightarrow \mu_{0}(\theta)^{+}} \rho^{B}(\beta, \mu) \equiv \rho_{\text {sup }}^{B}(\theta),
$$

where $\mu_{0}=\mu_{0}(\theta)$ is the inverse function of $\theta_{0}(\mu)$, and

$$
\lim _{\theta \rightarrow \theta_{0}(0)^{+}} \rho_{c}^{B}(\theta)<\lim _{\theta \rightarrow \theta_{0}(0)^{-}} \rho_{c}^{B}(\theta) .
$$

Thus the aim of the present paper is to study the thermodynamic properties of the WIBG in function of the total particle density $\rho$ to answer the question of its thermodynamic behaviour for the densities $\rho \geq \rho_{c}^{B}(\theta)$. Our main statements are formulated in the next Section II where we explicit the existence of a conventional (generalized) BoseEinstein condensation for $\mu=0$ and densities $\rho>\rho_{c}^{B}(\theta)$ which occurs after a non-conventional condensation (1.21) $[4,5]$ if $\theta \leq \theta_{0}(0)$, see Figure 1. Section III contains discussions and concluding remarks. Some technical statements are formulated in Appendix A.

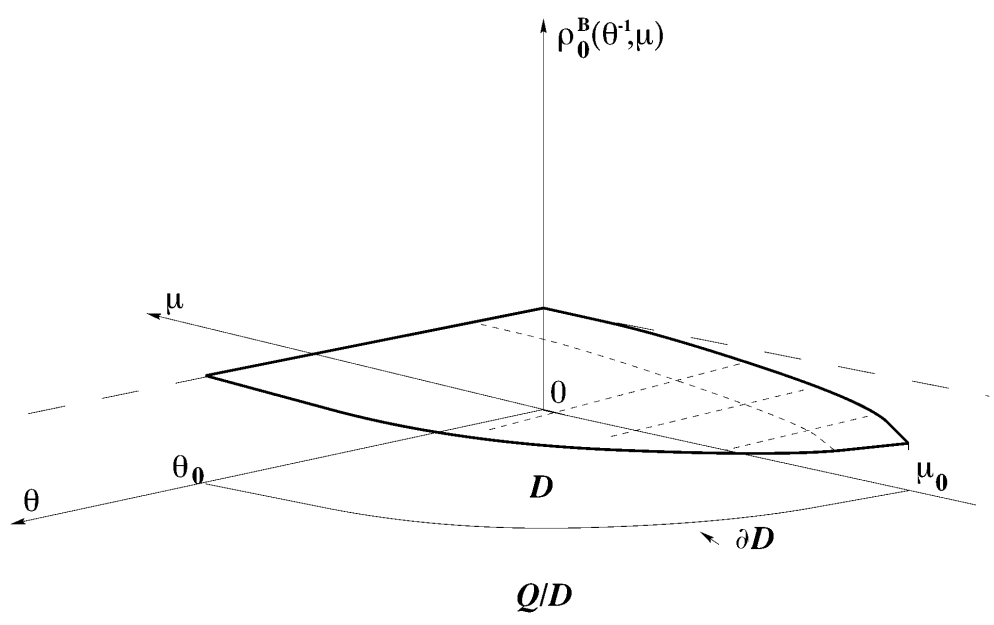

Fig. 1. Density of the non-conventional condensation in the Bogoliubov WIBG.

\section{BOSE-EINSTEIN CONDENSATION IN THE WIBG}

In this section we study the WIBG for temperature and the total particle density as given parameters.

Theorem 4. Let interaction (1.1) satisfies (A) and (B). Then there exists $\varepsilon_{\Lambda, 1}$ :

$$
\varepsilon_{\Lambda, 1} \in\left[\inf _{k \neq 0}\left[\varepsilon_{k}-\frac{v(k)}{2 V}\right], \widehat{\varepsilon}_{\Lambda, 1}=\inf _{k \neq 0} \varepsilon_{k}\right],
$$

such that for $\mu<\varepsilon_{\Lambda, 1}$

$$
p_{\Lambda}^{B}(\beta, \mu)<+\infty, \quad \omega_{\Lambda}^{B}\left(\frac{N_{\Lambda}}{V}\right)<+\infty
$$

and 


$$
\lim _{\mu \rightarrow \varepsilon_{\Lambda, 1}} p_{\Lambda}^{B}(\beta, \mu)=+\infty, \quad \lim _{\mu \rightarrow \varepsilon_{\Lambda, 1}} \omega_{\Lambda}^{B}\left(\frac{N_{\Lambda}}{V}\right)=+\infty
$$

Proof. Since $v(k)$ satisfies (A) and (B), by regrouping terms in (1.6), (1.7) one gets

$$
H_{\Lambda}^{B}=\widetilde{H}_{\Lambda}+\frac{v(0)}{V} a_{0}^{*} a_{0} \sum_{k \in \Lambda^{*}, k \neq 0} a_{k}^{*} a_{k}+\frac{1}{2 V} \sum_{k \in \Lambda^{*}, k \neq 0} v(k)\left(a_{0}^{*} a_{k}+a_{-k}^{*} a_{0}\right)^{*}\left(a_{0}^{*} a_{k}+a_{-k}^{*} a_{0}\right)
$$

where

$$
\tilde{H}_{\Lambda}=\sum_{k \in \Lambda^{*}, k \neq 0}\left(\varepsilon_{k}-\frac{v(k)}{2 V}\right) a_{k}^{*} a_{k}+\frac{v(0)}{2 V}\left(a_{0}^{*} a_{0}\right)^{2}-\frac{1}{2} \varphi(0) a_{0}^{*} a_{0}
$$

Thus from (2.3), (2.4) we obtain

$$
H_{\Lambda}^{B} \geq \widetilde{H}_{\Lambda}
$$

By straightforward calculations one gets

$$
p_{\Lambda}\left[\widetilde{H}_{\Lambda}\right]=\frac{1}{\beta V} \sum_{k \in \Lambda^{*}, k \neq 0} \ln \left\{1-e^{-\beta\left[\varepsilon_{k}-\left(\mu+\frac{\nu(k)}{2 V}\right)\right]}\right\}^{-1}+\frac{1}{\beta V} \ln \sum_{n_{0}=0}^{+\infty} e^{\beta V\left[\left(\mu+\frac{1}{2} \varphi(0)\right) \frac{n_{0}}{V}-\frac{\nu(0)}{2 V}\left[\frac{n_{0}}{V}\right]^{2}\right]},
$$

which together with (2.5) implies

$$
p_{\Lambda}^{B}(\beta, \mu) \leq p_{\Lambda}\left[\tilde{H}_{\Lambda}\right]<+\infty
$$

for $\mu<\inf _{k \neq 0}\left[\varepsilon_{k}-\frac{v(k)}{2 V}\right]$. Since

$$
\omega_{\Lambda}^{B}\left(\frac{N_{\Lambda}}{V}\right)=\partial_{\mu} p_{\Lambda}^{B}(\beta, \mu)
$$

by $(2.6)$ and by convexity of the pressure $p_{\Lambda}^{B}(\beta, \mu)$ in parameter $\mu$ we deduce that

$$
\omega_{\Lambda}^{B}\left(\frac{N_{\Lambda}}{V}\right)<+\infty
$$

for $\mu<\inf _{k \neq 0}\left[\varepsilon_{k}-\frac{v(k)}{2 V}\right]$. Moreover by the Bogoliubov inequality (see e.g. [8,9]), one gets:

$$
\frac{1}{V}\left\langle U_{\Lambda}\right\rangle_{H_{\Lambda}^{B}} \leq p_{\Lambda}\left[H_{\Lambda}^{B D}\right]-p_{\Lambda}\left[H_{\Lambda}^{B}\right] \leq \frac{1}{V}\left\langle U_{\Lambda}\right\rangle_{H_{\Lambda}^{B D}}
$$

where $H_{\Lambda}^{B D} \equiv T_{\Lambda}+U_{\Lambda}^{D}$ is the diagonal part of the Bogoliubov Hamiltonian with $T_{\Lambda}$ and $U_{\Lambda}^{D}$ defined respectively by (1.5) and (1.7). Since $\left\langle U_{\Lambda}\right\rangle_{H_{\Lambda}^{B D}}=0$, we deduce from
(2.7) that

$$
p_{\Lambda}^{B}(\beta, \mu) \geq p_{\Lambda}\left[H_{\Lambda}^{B D}\right]
$$

Combining this with the estimate (cf. [4,5])

$$
p_{\Lambda}\left[H_{\Lambda}^{B D}\right] \geq \frac{1}{\beta V} \sum_{k \in \Lambda^{*}, k \neq 0} \ln \left[\left(1-e^{\left[-\beta\left(\varepsilon_{k}-\mu\right)\right]}\right)^{-1}\right]
$$

we get

$$
\lim _{\mu \rightarrow \inf _{k \neq 0} \varepsilon_{k}} p_{\Lambda}\left[H_{\Lambda}^{B D}\right]=+\infty
$$

Therefore, by (2.6) and (2.8) we deduce that there exists $\varepsilon_{\Lambda, 1} \in\left[\inf _{k \neq 0}\left[\varepsilon_{k}-\frac{v(k)}{2 V}\right], \inf _{k \neq 0} \varepsilon_{k}\right]$ such that $p_{\Lambda}^{B}(\beta, \mu)$ and $\omega_{\Lambda}^{B}\left(\frac{N_{\Lambda}}{V}\right)$ are bounded for $\mu<\varepsilon_{\Lambda, 1}$ and

$$
\lim _{\mu \rightarrow \varepsilon_{\Lambda, 1}} p_{\Lambda}^{B}(\beta, \mu)=+\infty
$$

Notice that by convexity of $p_{\Lambda}^{B}(\beta, \mu)$ one gets

$$
\frac{p_{\Lambda}^{B}(\beta, \mu)-p_{\Lambda}^{B}(\beta, 0)}{\mu} \leq \partial_{\mu} p_{\Lambda}^{B}(\beta, \mu)=\omega_{\Lambda}^{B}\left(\frac{N_{\Lambda}}{V}\right)
$$


Then the limit (2.9) implies

$$
\lim _{\mu \rightarrow \varepsilon_{\Lambda, 1}} \omega_{\Lambda}^{B}\left(\frac{N_{\Lambda}}{V}\right)=+\infty
$$

which completes the proof of $(2.2)$.

Since the case of $\rho<\rho_{c}^{B}(\theta)(c f .(1.25),(1.26))$ has been already studied in Section I, see $(1.23),(1.24)$, below we consider the case $\rho \geq \rho_{c}^{B}(\theta)$.

Corollary 5. By Theorem f for any $\rho \geq \rho_{c}^{B}(\theta)$ there is a unique value of the chemical potential $\mu_{\Lambda}^{B}(\theta, \rho)<$ $\varepsilon_{\Lambda, 1}$ (notice that in general $\mu_{\Lambda}^{B}(\theta, \rho) \gtrless 0$ ) such that

$$
\omega_{\Lambda}^{B}\left(\frac{N_{\Lambda}}{V}\right)=\rho
$$

and

$$
\lim _{\Lambda} \mu_{\Lambda}^{B}\left(\theta, \rho \geq \rho_{c}^{B}(\theta)\right)=0 .
$$

From now on we put

$$
\left.\omega_{\Lambda, \rho}^{B}(-) \equiv \omega_{\Lambda}^{B}(-)\right|_{\mu=\mu_{\Lambda}^{B}(\theta, \rho)}
$$

According to $[4,5]$ the WIBG non-conventional condensation in the mode $k=0$ is saturated for $\mu \rightarrow 0^{-}$either by $|\widehat{c}(\beta, 0)|^{2}>0\left(\right.$ for $\left.\theta<\theta_{0}(0)\right)$, or by $|\widehat{c}(\beta, 0)|^{2}=0$ (for $\left.\theta>\theta_{0}(0)\right)$, see (1.21). Therefore, by (1.23)-(1.26) and Theorem 4 the saturation of the total particle density should imply the conventional Bose-Einstein condensation in modes next to $k=0$. For discussion of this phenomenon of two kinds of condensations in the framework of simple models see e.g. recent papers $[10,11]$.

To control the condensation in $k \neq 0$ we introduce an auxiliary Hamiltonian

$$
H_{\Lambda, \alpha}^{B}=H_{\Lambda}^{B}-\alpha \sum_{k \in \Lambda^{*}, a<\|k\|<b} a_{k}^{*} a_{k},
$$

for a fixed $a>0$ and $b>a>0$. Then we set

$$
p_{\Lambda}^{B}(\beta, \mu, \alpha) \equiv \frac{1}{\beta V} \ln \operatorname{Tr}_{\mathcal{F}_{\Lambda}} e^{-\beta H_{\Lambda, \alpha}^{B}(\mu)},
$$

and

$$
\omega_{\Lambda}^{B, \alpha}(-) \equiv\langle-\rangle_{H_{\Lambda, \alpha}^{B}}(\beta, \mu)
$$

for the grand-canonical Gibbs state corresponding to $H_{\Lambda, \alpha}^{B}(\mu)$.

Recall that $\mu_{0}(\theta)$ is the function (inverse to $\theta_{0}(\mu)$ ) which defines a borderline of domain $D$, see (1.19).

Proposition 6. $[4,5]$ Let $\alpha \in[-\delta, \delta]$ where $0 \leq \delta \leq$ $\varepsilon_{a} / 2$ and $\varepsilon_{a}=\inf _{\|k\| \geq a} \varepsilon_{k}$. Then there exists a domain $D_{\delta} \subset D$ :

$$
D_{\delta} \equiv\left\{(\theta, \mu): \mu_{0}<\mu_{0}(\delta) \leq \mu \leq 0,0 \leq \theta \leq \theta_{0}(\mu, \delta)<\theta_{0}(\mu)\right\}
$$

such that

$$
\left|p_{\Lambda}^{B}(\beta, \mu, \alpha)-\sup _{c \in \mathbb{C}} \tilde{p}_{\Lambda}^{B}\left(\beta, \mu, \alpha ; c^{\#}\right)\right| \leq \frac{K(\delta)}{\sqrt{V}}
$$

for $V$ sufficiently large, uniformly in $\alpha \in[-\delta, \delta]$ and for:

(i) $(\theta, \mu) \in D_{\delta}$, if $\mu_{\Lambda}^{B}\left(\theta, \rho \geq \rho_{c}^{B}(\theta)\right) \leq 0$;

(ii) $(\theta, \mu) \in D_{\delta} \cup\left\{(\theta, \mu): 0 \leq \mu \leq \mu_{\Lambda}^{B}\left(\theta, \rho \geq \rho_{c}^{B}(\theta)\right), 0 \leq \theta \leq \theta_{0}(\mu=0, \delta)\right\}, \quad$ if $\quad \mu_{\Lambda}^{B}\left(\theta, \rho \geq \rho_{c}^{B}(\theta)\right) \geq 0$.

Proof. The existence of the domain $D_{\delta}$ follows from the proof of Theorem 3.14 [5]. This means that the estimate (2.15) is stable with respect to local perturbations of the free-particle spectrum: $\varepsilon_{k} \rightarrow \varepsilon_{k}-\alpha \chi(a, b)(\|k\|)$ for $|\alpha| \leq \delta \leq$ $\varepsilon_{a} / 2$ in a reduced domain $D_{\delta} \subset D$. Here $\chi_{(a, b)}(\|k\|)$ is the characteristic function of $(a, b) \subset \mathbb{R}$. Extension in $(2.16)$ (cf. Corollary 5) is due to continuity of the pressure $p_{\Lambda}^{B}(\beta, \mu, \alpha)$ and the trial pressure $\tilde{p}_{\Lambda}^{B}\left(\beta, \mu, \alpha ; c^{\#}\right)$ in parameters $\alpha \in[-\delta, \delta]$ and $\mu \leq \mu_{\Lambda}^{B}\left(\theta, \rho \geq \rho_{c}^{B}(\theta)\right)$, see $(2.11)$.

Corollary 7. Let $\rho \geq \rho_{c}^{B}(\theta)$, see (1.25), (1.26). Then for $\theta<\theta_{0}(0)$ one has

$$
\lim _{\Lambda} \frac{1}{V} \sum_{k \in \Lambda^{*}, a<\|k\|<b} \omega_{\Lambda, \rho}^{B}\left(N_{k}\right)=\left.\frac{1}{(2 \pi)^{3}} \int_{a<\|k\|<b}\left[\frac{f_{k}}{E_{k}}\left(e^{\beta E_{k}}-1\right)^{-1}+\frac{h_{k}^{2}}{2 E_{k}\left(f_{k}+E_{k}\right)}\right] d^{3} k\right|_{\substack{c=\hat{c}(\beta, 0) \\ \mu=0}},
$$


whereas for $\theta>\theta_{0}(0)$ we have

$$
\lim _{\Lambda} \frac{1}{V} \sum_{k \in \Lambda^{*}, a<\|k\|<b} \omega_{\Lambda, \rho}^{B}\left(N_{k}\right)=\frac{1}{(2 \pi)^{3}} \int_{a<\|k\|<b}\left(e^{\beta \varepsilon_{k}}-1\right)^{-1} d^{3} k
$$

Proof. Consider the sequence of functions $\left\{p_{\Lambda}^{B}\left(\beta, \mu_{\Lambda}^{B}(\theta, \rho), \alpha\right)\right\}_{\Lambda}(2.13)$, where chemical potential is defined by (2.10), (2.11) and $\alpha \in[-\delta, \delta]$. Since by $(2.13)$

$$
\partial_{\alpha} p_{\Lambda}^{B}\left(\beta, \mu_{\Lambda}^{B}(\theta, \rho), \alpha\right)=\frac{1}{V} \sum_{k \in \Lambda^{*}, a<\|k\|<b} \omega_{\Lambda, \rho}^{B, \alpha}\left(N_{k}\right)
$$

and $\left\{p_{\Lambda}^{B}\left(\beta, \mu_{\Lambda}^{B}(\theta, \rho), \alpha\right)\right\}_{\Lambda}$ are convex functions of $\alpha \in[-\delta, \delta]$, Proposition 6 and the Griffiths lemma [6,7] imply

$$
\lim _{\Lambda} \partial_{\alpha} p_{\Lambda}^{B}\left(\beta, \mu_{\Lambda}^{B}(\theta, \rho), \alpha\right)=\lim _{\Lambda} \frac{1}{V} \sum_{k \in \Lambda^{*}, a<\|k\|<b} \omega_{\Lambda, \rho}^{B, \alpha}\left(N_{k}\right)=\partial_{\alpha} \lim _{\Lambda} \sup _{c \in \mathbb{C}} \tilde{p}_{\Lambda}^{B}\left(\beta, \mu_{\Lambda}^{B}(\theta, \rho), \alpha ; c^{\#}\right),
$$

for $\alpha \in[-\delta, \delta]$. Therefore, by explicit calculations in the right-hand side of (2.20) (cf. (1.10)-(1.12)) we obtain for $\alpha=0$ equalities (2.17) and (2.18).

Remark 1. Notice that the expectation values $\omega_{\Lambda}^{B}\left(N_{k}\right)=\left\langle N_{k}\right\rangle_{H_{\Lambda}^{B}}(\beta, \mu)$ (and similar $\omega_{\Lambda, \rho}^{B}\left(N_{k}\right)=$ $\left.\left\langle N_{k}\right\rangle_{H_{\Lambda}^{B}}\left(\beta, \mu_{\Lambda}^{B}(\theta, \rho)\right)\right)$ are defined on the discrete set $\Lambda^{*}$. Below we denote by $\left\{\omega_{\Lambda}^{B}\left(N_{k}\right)\right\}_{k \in \mathbb{R}^{3}}$ a continuous interpolation of these values from the set $\Lambda^{*}$ to $\mathbb{R}^{3}$.

Now we are in position to prove the main statement of this section about the Bose-Einstein condensation manifested by the WIBG for large densities $\rho$ at fixed temperature $\theta=\beta^{-1}$.

Theorem 8. For $\rho>\rho_{c}^{B}(\theta)$ we have that

(i)

$$
\lim _{\Lambda} \omega_{\Lambda, \rho}^{B}\left(\frac{a_{0}^{*} a_{0}}{V}\right)=\left\{\begin{array}{c}
|\widehat{c}(\beta, 0)|^{2}, \theta<\theta_{0}(0) \\
0, \theta>\theta_{0}(0)
\end{array}\right\}
$$

(ii) for any $k \in \Lambda^{*}$, such that $\|k\|>\frac{2 \pi}{L}$,

$$
\lim _{\Lambda} \omega_{\Lambda, \rho}^{B}\left(\frac{N_{k}}{V}\right)=0
$$

(iii) for $\theta<\theta_{0}(0)$ and for all $k \in \Lambda^{*}$, such that $\|k\|>\delta>0$

$$
\begin{aligned}
\lim _{\Lambda} \omega_{\Lambda, \rho}^{B}\left(N_{k}\right) & =\left[\frac{f_{k}}{E_{k}}\left(e^{\beta E_{k}}-1\right)^{-1}\right. \\
& \left.+\frac{h_{k}^{2}}{2 E_{k}\left(f_{k}+E_{k}\right)}\right]_{\substack{c=\hat{c}(\beta, 0) \\
\mu=0}}
\end{aligned}
$$

whereas for $\theta>\theta_{0}(0)$

$$
\lim _{\Lambda} \omega_{\Lambda, \rho}^{B}\left(N_{k}\right)=\frac{1}{e^{\beta \varepsilon_{k}}-1}
$$

(iv) the double limit

$$
\lim _{\delta \rightarrow 0^{+}} \lim _{\Lambda} \frac{1}{V} \sum_{\left\{k \in \Lambda^{*}, 0<\|k\| \leq \delta\right\}} \omega_{\Lambda, \rho}^{B}\left(N_{k}\right)=\rho-\rho_{c}^{B}(\theta)
$$

which means that the WIBG manifests a conventional generalized Bose-Einstein condensation in the $2 d$ modes next to the zero-mode due to particle density saturation.

Proof. (i) Since by (2.11) we have

$$
\lim _{\Lambda} \mu_{\Lambda}^{B}(\theta, \rho)=0
$$

the thermodynamic limit (2.21) results from Theorem 4.4 and Corollary 4.8 of [5], see also (1.21) for $\mu=0$.

(ii) Since $\|k\|>\frac{2 \pi}{L}$ and $\Lambda=L \times L \times L$, which excludes a generalized Bose-Einstein condensation due to anisotropy [12], the thermodynamic limit (2.22) follows from Lemma 10.

(iii) Let us consider $g_{\theta}(k)$ defined for $k \in \mathbb{R}^{3},\|k\|>$ $\delta>0$ by

$$
g_{\theta}(k) \equiv \lim _{\Lambda} \omega_{\Lambda, \rho}^{B}\left(N_{k}\right)
$$

where (cf. $(2.12))$ the state $\omega_{\Lambda, \rho}^{B}(-)$ stands for $\omega_{\Lambda}^{B}(-)$ with $\mu=\mu_{\Lambda}^{B}(\theta, \rho)$. Notice that by Lemma 10 and the fact that 


$$
\mu_{\Lambda}^{B}(\theta, \rho)<\varepsilon_{\Lambda, 1}<\inf _{k \neq 0} \varepsilon_{k}=\varepsilon_{\|k\|=\frac{2 \pi}{L}}
$$

the thermodynamic limit (2.27) exists and it is informally bounded for $\|k\|>\delta>0$. Moreover, for any interval $(a>\delta, b)$ we have

$$
\begin{aligned}
& \lim _{\Lambda} \frac{1}{V} \sum_{k \in \Lambda^{*},\|k\| \in(a, b)} \omega_{\Lambda, \rho}^{B}\left(N_{k}\right) \\
& =\frac{1}{(2 \pi)^{3}} \int_{\|k\|>\delta} g_{\theta}(k) \chi_{(a, b)}(\|k\|) d^{3} k,
\end{aligned}
$$

where $\chi_{(a, b)}(\|k\|)$ is the characteristic function of $(a, b)$. Then Corollary 7 implies that

$$
\begin{aligned}
& \frac{1}{(2 \pi)^{3}} \int_{\|k\|>\delta} g_{\theta}(k) \chi_{(a, b)}(\|k\|) d^{3} k \\
& =\frac{1}{(2 \pi)^{3}} \int_{\|k\|>\delta} f_{\theta}(k) \chi_{(a, b)}(\|k\|) d^{3} k,
\end{aligned}
$$
interval $(a>\delta, b) \subset \mathbb{R}$ one gets

$$
g_{\theta}(k)=f_{\theta}(k), k \in \mathbb{R}^{3},\|k\|>\delta>0
$$

for $\theta<\theta_{0}(0)$ and

$$
f_{\theta}(k) \equiv \frac{1}{(2 \pi)^{3}}\left(e^{\beta \varepsilon_{k}}-1\right)^{-1}
$$

for $\theta>\theta_{0}(0)$. Since the relation (2.28) is valid for any

from which by $(2.27),(2.29)$ and $(2.30)$ we deduce $(2.23)$ and (2.24).

(iv) Since the total density $\rho$ is fixed, we have

$$
\frac{1}{V} \sum_{\left\{k \in \Lambda^{*}, 0<\|\mid k\| \leq \delta\right\}} \omega_{\Lambda, \rho}^{B}\left(N_{k}\right)=\rho-\omega_{\Lambda, \rho}^{B}\left(\frac{a_{0}^{*} a_{0}}{V}\right)-\frac{1}{V} \sum_{\left\{k \in \Lambda^{*}:\|k\|>\delta\right\}} \omega_{\Lambda, \rho}^{B}\left(N_{k}\right)
$$

Using Corollary 7 for $a=\delta$ and $b \rightarrow+\infty$ we obtain for $\theta<\theta_{0}(0)$

$$
\lim _{\Lambda} \frac{1}{V} \sum_{\left\{k \in \Lambda^{*}:\|k\|>\delta\right\}} \omega_{\Lambda, \rho}^{B}\left(N_{k}\right)=\frac{1}{(2 \pi)^{3}}\left[\int_{\|k\|>\delta} \frac{f_{k}}{E_{k}}\left(e^{\beta E_{k}}-1\right)^{-1}+\frac{h_{k}^{2}}{2 E_{k}\left(f_{k}+E_{k}\right)} d^{3} k\right]_{\substack{c=\hat{c}(\beta, 0) \\ \mu=0}},
$$

and for $\theta>\theta_{0}(0)$

$$
\lim _{\Lambda} \frac{1}{V} \sum_{k \in \Lambda^{*},\|k\|>\delta} \omega_{\Lambda, \rho}^{B}\left(N_{k}\right)=\frac{1}{(2 \pi)^{3}} \int_{\|k\|>\delta}\left(e^{\beta \varepsilon_{k}}-1\right)^{-1} d^{3} k
$$

Then, from (1.25), (1.26), (2.21), (2.31)-(2.33) we finally deduce $(2.25)$ by taking the limit $\delta \rightarrow 0^{+}$.

Therefore, according to (2.25) and in a close similarity to [11] for $\theta>\theta_{0}(0)$ and $\rho>\rho_{c}^{B}(\theta)$ the WIBG manifests only one kind of condensation, namely a conventional Bose-Einstein condensation which occurs in the mode $k \neq 0$, whereas for $\theta<\theta_{0}(0)$ it manifests for $\rho>\rho_{c}^{B}(\theta)$ this kind of condensation as a second stage after the nonconventional Bose condensation $|\hat{c}(\beta, 0)|^{2}$, see $(2.21)$.

Remark 2. Similar to the model of ref. [11] in domain: $\theta<\theta_{0}(0), \rho>\rho_{c}^{B}(\theta)$, we have the coexistence of two kinds of condensations:

- the non-conventional one which starts when $\rho>$ $\rho_{\text {sup }}^{B}(\theta)\left(\rho \leq \rho_{c}^{B}(\theta)\right)$, see (1.25)-(1.27),

- and the conventional Bose-Einstein condensation when $\rho>\rho_{c}^{B}(\theta)$.

Remark 3. Before we classify this latter condensation we remind to the readers about the convenience of the nomenclature of conventional (generalized) BoseEinstein condensations according to [12,13]:

- a condensation is called of type I when a finite number of levels is macroscopically occupied; 
- it is of type II when an infinite number of levels is macroscopically occupied;

- it is called of type III, or the non-extensive condensation, when no levels are macroscopically occupied whereas one has

$$
\lim _{\delta \rightarrow 0^{+}} \lim _{\Lambda} \frac{1}{V} \sum_{\left\{k \in \Lambda^{*}, 0<\|k\| \leq \delta\right\}}\left\langle N_{k}\right\rangle=\rho-\rho_{c}(\theta) .
$$

Paper [12] demonstrates that these three kinds of conventional condensations can be realized for the case of the $\mathrm{PBG}$ in an anisotropic box $\Lambda \subset \mathbb{R}^{3}$ with volume the $V=|\Lambda|$ and the Dirichlet boundary conditions, i.e. in a box $\Lambda$ with $L_{x}=V^{\alpha_{x}}, L_{y}=V^{\alpha_{y}}$ and $L_{z}=V^{\alpha_{z}}$ for $\alpha_{x}+\alpha_{y}+\alpha_{z}=1$ and $\alpha_{x} \leq \alpha_{y} \leq \alpha_{z}$. At fixed temperature and for sufficiently large density $\rho$, we have a condensation of the type $I$ in the fundamental mode $k=\left(\frac{2 \pi}{L_{x}}, \frac{2 \pi}{L_{y}}, \frac{2 \pi}{L_{z}}\right)$ if $\alpha_{z}<1 / 2$ whereas for $\alpha_{z}=1 / 2$ one gets a condensation of the type II characterized by a macroscopic occupation of all modes $k=\left(\frac{2 \pi}{L_{x}}, \frac{2 \pi}{L_{y}}, \frac{2 \pi n}{L_{Z}}\right)$, $n \in \mathbb{N}$ and for $\alpha_{z}>1 / 2$ one obtains a condensation of the type III. In $[14,15]$ it was shown that type III conden- sation can be provoked in the PBG by a weak external potential or (see [13,16]) by a specific choice of boundary conditions and geometry. Another example of the nonextensive condensation is given in $[10,11]$ for bosons in an isotropic box $\Lambda$ with interactions which spread out the conventional condensation of the type I into a conventional condensation of the type III.

Therefore, from (2.22) and (2.25) we can deduce only that the conventional condensation in the WIBG can be either a condensation of type I in modes $\|k\|=2 \pi / L$, or a condensation of the type III if modes $\|k\|=2 \pi / L$ are not macroscopically occupied, or finally a combination of the non-extensive condensation with a condensation of the type $\mathrm{I}$ in the modes $\|k\|=2 \pi / L$.

Corollary 9. In fact, for $\rho>\rho_{c}^{B}(\theta)$ the generalized (conventional) condensation (2.25) is a condensation of the type $I$ in the first $2 d$ modes next to the zero-mode $k=0$, i.e.

$$
\lim _{\Lambda} \frac{1}{V} \sum_{\left\{k \in \Lambda^{*},\|k\|=\frac{2 \pi}{L}\right\}} \omega_{\Lambda, \rho}^{B}\left(a_{k}^{*} a_{k}\right)=\rho-\rho_{c}^{B}(\theta)
$$

Proof. Since for $\delta>0$

$$
\frac{1}{V} \sum_{\left\{k \in \Lambda^{*},\|k\|=\frac{2 \pi}{L}\right\}} \omega_{\Lambda, \rho}^{B}\left(N_{k}\right)=\rho-\omega_{\Lambda, \rho}^{B}\left(\frac{a_{0}^{*} a_{0}}{V}\right)-\frac{1}{V} \sum_{\left\{k \in \Lambda^{*}, \frac{2 \pi}{L}<\|k\|<\delta\right\}} \omega_{\Lambda, \rho}^{B}\left(N_{k}\right)-\frac{1}{V} \sum_{\left\{k \in \Lambda^{*}:\|k\| \geq \delta\right\}} \omega_{\Lambda, \rho}^{B}\left(N_{k}\right)
$$

using Lemma 1 we find that

$$
\begin{aligned}
\frac{1}{V} \sum_{\left\{k \in \Lambda^{*},\|k\|=\frac{2 \pi}{L}\right\}} \omega_{\Lambda, \rho}^{B}\left(N_{k}\right) & \geq \rho-\frac{1}{V} \sum_{\left\{k \in \Lambda^{*}, \frac{2 \pi}{L}<\|k\|<\delta\right\}} \frac{1}{e^{B_{k}\left(\mu_{\Lambda}^{B}(\theta, \rho)\right)}-1} \\
& -\omega_{\Lambda, \rho}^{B}\left(\frac{a_{0}^{*} a_{0}}{V}\right)\left[1+\frac{\beta}{2 V} \sum_{\left\{k \in \Lambda^{*}, \frac{2 \pi}{L}<\|k\|<\delta\right\}} \frac{v(k)}{\left.1-e^{-B_{k}\left(\mu_{\Lambda}^{B}(\theta, \rho)\right)}\right]}\right. \\
& -\frac{1}{V} \sum_{\left\{k \in \Lambda^{*}:\|k\| \geq \delta\right\}} \omega_{\Lambda, \rho}^{B}\left(N_{k}\right),
\end{aligned}
$$

with $B_{k}\left(\mu_{\Lambda}^{B}(\theta, \rho)\right)$ defined by $(4.2)$. Since by Theorem 1 one gets

$$
\mu_{\Lambda}^{B}(\theta, \rho)<\varepsilon_{\Lambda, 1}<\inf _{k \neq 0} \varepsilon_{k}=\varepsilon_{\|k\|=\frac{2 \pi}{L}},
$$

from $(1.25),(1.26),(2.32)$ we deduce

$$
\lim _{\Lambda} \frac{1}{V} \sum_{\left\{k \in \Lambda^{*},\|k\|=\frac{2 \pi}{L}\right\}} \omega_{\Lambda, \rho}^{B}\left(a_{k}^{*} a_{k}\right) \geq \rho-\rho_{c}^{B}(\theta)
$$

by taking the limit $\delta \rightarrow 0^{+}$in the right-hand side of (2.35) after the thermodynamic limit. Therefore, combining the 
inequality

$$
\begin{aligned}
& \lim _{\Lambda} \frac{1}{V} \sum_{\left\{k \in \Lambda^{*},\|k\|=\frac{2 \pi}{L}\right\}} \omega_{\Lambda, \rho}^{B}\left(N_{k}\right) \\
& \leq \lim _{\Lambda} \frac{1}{V} \sum_{\left\{k \in \Lambda^{*}, 0<\|k\|<\delta\right\}} \omega_{\Lambda, \rho}^{B}\left(N_{k}\right)
\end{aligned}
$$

with Theorem 5 (cf. (2.25)) and (2.36)), we obtain (2.34).

Therefore, for a fixed temperature $\theta$ and a fixed total particle density $\rho$, we obtain three types of thermodynamic behaviour of the WIBG for $\theta<\theta_{0}(0)$ :

(i) for $\rho \leq \rho_{\text {inf }}^{B}(\theta)$, there is no condensation;

(ii) for $\rho_{\text {sup }}^{B}(\theta) \leq \rho \leq \rho_{c}^{B}(\theta)$, there is a nonconventional (dynamical) condensation (1.21) in the mode $k=0$ due to non-diagonal interaction in the Bogoliubov Hamiltonian, see Figure 1 and $[4,5,17]$;

(iii) for $\rho_{c}^{B}(\theta) \leq \rho$, there is a second kind of condensation: the conventional type I Bose-Einstein condensation which occurs after the non-conventional one due to the standard mechanism of the total particle density saturation (Corollary 9).

If $\theta \geq \theta_{0}(0)$, there are only two types of thermodynamic behaviour: they correspond to $\rho \leq \rho_{c}^{B}(\theta)$ with no condensation and to $\rho_{c}^{B}(\theta) \leq \rho$ with a conventional condensation as in (iii). Hence, for $\theta>\theta_{0}(0)$ the condensation in the WIBG coincides with type I generalized Bose-Einstein condensation in the PBG with excluded mode $k=0$, see Theorem 8 (iii) and [18].

\section{CONCLUSION}

Papers [4,5] have already discussed the existence of a non-conventional condensation of bosons for $k=0$, for negative $\mu$ and $\theta<\theta_{0}(0)$. The physical reason of this non-conventional (or dynamical) condensation is an effective attraction between bosons in the mode $k=0$ [17]:

$$
-\left\{\frac{1}{V^{2}} \sum_{k \in \Lambda^{*}, k \neq 0} \frac{[v(k)]^{2}}{4 \varepsilon_{k}}\right\} a_{0}^{*^{2}} a_{0}^{2}
$$

which has to dominate the direct repulsion in (1.7):

$$
\frac{v(0)}{2 V} a_{0}^{*^{2}} a_{0}^{2}
$$

to ensure this new kind of condensation, see condition (C) (1.17) and discussions in [17]. However, for fixed temperature $\theta$ and total particle density $\rho$ the present paper indicates the possibility of a conventional condensation: a generalized Bose-Einstein condensation of the type $I$ in the first $2 d$ modes next to the zero-mode $k=0$. This second kind of condensation appears only for high densities $\rho \geq \rho_{c}^{B}(\theta)$ due to the standard mechanism of the total particle density saturation, see Corollary 9 .

Therefore, combining [4,5] with Section II for $\theta<$ $\theta_{0}(0)$ we obtain for the WIBG three types of thermodynamic behaviour:

(i) for $\rho \leq \rho_{\text {inf }}^{B}(\theta)$, there is no condensation;

(ii) for $\rho_{\text {sup }}^{\bar{B}}(\theta) \leq \rho \leq \rho_{c}^{B}(\theta)$, a non-conventional (dynamical) condensation (1.21) appears in the mode $k=0$;

(iii) for $\rho_{c}^{B}(\theta) \leq \rho$, the WIBG manifests a conventional Bose-Einstein condensation of the type I (Corollary 6). Therefore, two kinds of condensation coexist.

For $\theta<\theta_{0}(0)$, the thermodynamic behaviour of the WIBG is related to the two recent models [11] defined respectively by Hamiltonians

$$
H_{\Lambda}^{0} \equiv T_{\Lambda}+U_{\Lambda}^{0}
$$

and

$$
H_{\Lambda}=H_{\Lambda}^{0}+U_{\Lambda}
$$

where

$$
\begin{aligned}
T_{\Lambda} & =\sum_{k \in \Lambda^{*} \backslash\{0\}} \varepsilon_{k} a_{k}^{*} a_{k}, \quad \varepsilon_{k} \neq 0=\hbar^{2} k^{2} / 2 m, \\
U_{\Lambda}^{0} & =\varepsilon_{0} a_{0}^{*} a_{0}+\frac{g_{0}}{V} a_{0}^{*} a_{0}^{*} a_{0} a_{0}, \varepsilon_{0} \in \mathbb{R}^{1}, g_{0}>0, \\
U_{\Lambda} & =\frac{1}{V} \sum_{k \in \Lambda^{*}, k \neq 0} g_{k}(V) a_{k}^{*} a_{k}^{*} a_{k} a_{k},
\end{aligned}
$$

with $0<g_{k}(V) \leq \gamma_{k} V^{\alpha_{k}}$ for $k \in \Lambda^{*} \backslash\{0\}, \alpha_{k} \leq \alpha_{+}<1$ and $0<\gamma_{k} \leq \gamma_{+}$. Notice that in these models, $\varepsilon_{0} \in \mathbb{R}^{1}$ is not equal to $\varepsilon_{\|k\|=0}=0$. Paper [11] shows the possibility of coexistence of two kinds of Bose condensations equally for models (3.2) and (3.3). In particular the WIBG is close to model (3.2) for $\theta<\theta_{0}(0)$ in the sense that the Bose gas (3.2) manifests the same three types of thermodynamic behaviour $(i)-($ iii $)$ as above but there is no limiting temperature $\theta_{0}(0)$ and no discontinuity of the condensate and the total particle density. The peculiarity of model (3.3) is that under conditions $g_{k \neq 0}(V) \geq g_{-}>0$ or $\inf _{\|k\|<\delta_{0}, V} g_{k}(V)>0$ in a band $\delta_{0}>0$, the direct repulsion $U_{\Lambda}(3.4)$ spreads out the conventional BoseEinstein condensation, originally of the type I in modes $\|k\|=\frac{2 \pi}{L}$, into a conventional Bose-Einstein condensation of the type III (cf. $[10,11]$ ). Notice that the conventional Bose-Einstein condensation persists in the model (3.3) even if for $k \in \Lambda^{*} \backslash\{0\} g_{k}(V)=\gamma_{k} V^{\alpha_{k}} \stackrel{V \rightarrow+\infty}{\rightarrow}+\infty$ $\left(\alpha_{k} \leq \alpha_{+}<1\right)$ which is similar to the WIBG where in the effective two-bosons repulsion for $k, q \neq 0$

$$
g_{\Lambda, k q} a_{k}^{*} a_{-k}^{*} a_{-q} a_{q}
$$

the "form-factor" $g_{\Lambda, k q}>0$ diverges with volume as $V^{2 / 3}$, see [17]. However, an important difference is that this effective interaction (which is due to non-diagonal term (1.7)) does not able to spread out the Bose-Einstein 
condensation into the type III as in the model (3.3) for the WIBG: it rests as a condensation of the type $I$.

For $\theta \geq \theta_{0}(0)$, there are only two types of thermodynamic behaviour: they correspond to the domain $\rho \leq$ $\rho_{c}^{B}(\theta)$ where there is no condensation and to $\rho_{c}^{B}(\theta) \leq \bar{\rho}$ where we have a conventional condensation as in $(\overline{i i i})$. Hence, for $\theta>\theta_{0}(0)$ the condensation in the WIBG coincides with the type I generalized Bose-Einstein condensation in the PBG with excluded mode $k=0$, see (iii) in Theorem 8 and [18].

Notice that one of the possibility to correct the instabilities of the WIBG for $\mu>0$ (originally discovered in [21]) would be to add to $H_{\Lambda}^{B}(1.4)$ the "forwardscattering" repulsive interaction between particles next to the zero-mode $k=0$ :

$$
H_{\Lambda}=H_{\Lambda}^{B}+\frac{v(0)}{2 V} \sum_{k, q \in \Lambda^{*} \backslash\{0\}} a_{k}^{*} a_{q}^{*} a_{q} a_{k}
$$

Paper [21] proposes to use the superstable Hamiltonian (3.5) to extract the gapless spectrum by doing the Bogoliubov approximation (see Definition 1 ) only in the operator $H_{\Lambda}^{B}-v(0) a_{0}^{*^{2}} a_{0}^{2} / 2 V$ (see also [22,23]). In fact the problem of the thermodynamics and the gapless spectrum for stabilized WIBG models is rather delicate, see discussions in [21-23]. The reason is that the interaction in the WIBG is in fact a long-range one, which implies the appearance of the gap when one has the nonconventional condensation in the zero-mode, see [5].

\section{ACKNOWLEDGMENTS}

The main ideas of this paper were formulated in numerous discussion with N. Angelescu, J.-B. Bru and A. Verbeure. I would like to thank them for these valuable discussions.

\section{APPENDIX A}

\section{Lemma 10.}

Let $\|k\|>2 \pi / L$. Then for the Gibbs state $\omega_{\Lambda, \rho}^{B}(-)$ we have:

$$
\omega_{\Lambda, \rho}^{B}\left(N_{k}\right) \leq \frac{1}{e^{B_{k}\left(\mu_{\Lambda}^{B}(\theta, \rho)\right)}-1}+\beta \frac{v(k)}{2 V} \frac{\omega_{\Lambda, \rho}^{B}\left(a_{0}^{*} a_{0}\right)}{1-e^{-B_{k}\left(\mu_{\Lambda}^{B}(\theta, \rho)\right)}},
$$

with

$$
B_{k}\left(\mu=\mu_{\Lambda}^{B}(\theta, \rho)\right) \equiv \beta\left[\varepsilon_{k}-\mu_{\Lambda}^{B}(\theta, \rho)-\frac{v(k)}{2 V}\right]
$$

Proof. By the correlation inequalities for the Gibbs state $\omega_{\Lambda}^{B}(-) \equiv\langle-\rangle_{H_{\Lambda}^{B}}(\beta, \mu)$ (see $\left.[19,20]\right)$ :

$$
\beta \omega_{\Lambda}^{B}\left(X^{*}\left[H_{\Lambda}^{B}(\mu), X\right]\right) \geq \omega_{\Lambda}^{B}\left(X^{*} X\right) \ln \frac{\omega_{\Lambda}^{B}\left(X^{*} X\right)}{\omega_{\Lambda}^{B}\left(X X^{*}\right)}
$$

where $X$ is an observable from the domain of the commutator $\left[H_{\Lambda}^{B}(\mu),.\right]$, we deduce

$$
\beta \omega_{\Lambda}^{B}\left(a_{k}^{*}\left[H_{\Lambda}^{B}(\mu), a_{k}\right]\right) \geq \omega_{\Lambda}^{B}\left(N_{k}\right) \ln \frac{\omega_{\Lambda}^{B}\left(N_{k}\right)}{\omega_{\Lambda}^{B}\left(N_{k}\right)+1}
$$

for $X=a_{k}$. Since for $\|k\|>2 \pi / L$

$$
\left[H_{\Lambda}^{B}(\mu), a_{k}\right]=-\left(\varepsilon_{k}-\mu-[v(0)+v(k)] \frac{a_{0}^{*} a_{0}}{V}\right) a_{k}-\frac{v(k)}{V} a_{0}^{2} a_{-k}^{*}
$$

one gets for $\mu=\mu_{\Lambda}^{B}(\theta, \rho)$ that

$$
\begin{aligned}
\omega_{\Lambda, \rho}^{B}\left(a_{k}^{*}\left[H_{\Lambda}^{B}\left(\mu_{\Lambda}^{B}(\theta, \rho)\right), a_{k}\right]\right) & =-\left[\varepsilon_{k}-\mu_{\Lambda}^{B}(\theta, \rho)\right] \omega_{\Lambda, \rho}^{B}\left(N_{k}\right)-[v(0)+v(k)] \frac{\omega_{\Lambda, \rho}^{B}\left(a_{0}^{*} a_{0} N_{k}\right)}{V} \\
& -v(k) \frac{\omega_{\Lambda, \rho}^{B}\left(a_{0}^{2} a_{k}^{*} a_{-k}^{*}\right)}{V}
\end{aligned}
$$

Notice that $\omega_{\Lambda, \rho}^{B}\left(a_{k}^{*}\left[H_{\Lambda}^{B}\left(\mu_{\Lambda}^{B}(\theta, \rho)\right), a_{k}\right]\right) \in \mathbb{R}$, then by $(4.5) \omega_{\Lambda, \rho}^{B}\left(a_{0}^{2} a_{k}^{*} a_{-k}^{*}\right) \in \mathbb{R}$. Therefore,

$$
2 \omega_{\Lambda, \rho}^{B}\left(a_{0}^{2} a_{k}^{*} a_{-k}^{*}\right)=\omega_{\Lambda, \rho}^{B}\left(a_{0}^{2} a_{k}^{*} a_{-k}^{*}\right)+\omega_{\Lambda, \rho}^{B}\left(a_{k} a_{-k} a_{0}^{* 2}\right)
$$

Moreover, since the functions $\varepsilon_{k}$ and $v(k)$ are even, we have 


$$
\omega_{\Lambda, \rho}^{B}\left(a_{0}^{*} a_{0} N_{k}\right)=\omega_{\Lambda, \rho}^{B}\left(a_{0}^{*} a_{0} N_{-k}\right)
$$

Thus (4.5)-(4.7) imply

$$
\begin{aligned}
\omega_{\Lambda, \rho}^{B}\left(a_{k}^{*}\left[H_{\Lambda}^{B}\left(\mu_{\Lambda}^{B}(\theta, \rho)\right), a_{k}\right]\right) & =-\left[\varepsilon_{k}-\mu_{\Lambda}^{B}(\theta, \rho)\right] \omega_{\Lambda, \rho}^{B}\left(a_{k}^{*} a_{k}\right)-\frac{v(k)}{2 V} \omega_{\Lambda, \rho}^{B}\left(a_{0}^{2} a_{k}^{*} a_{-k}^{*}+a_{0}^{* 2} a_{k} a_{-k}\right) \\
& -\frac{[v(0)+v(k)]}{2 V} \omega_{\Lambda, \rho}^{B}\left(a_{0}^{*} a_{0} N_{k}+a_{0}^{*} a_{0} N_{-k}\right) .
\end{aligned}
$$

Now applying the identity

$$
a_{0}^{2} a_{k}^{*} a_{-k}^{*}+a_{0}^{* 2} a_{k} a_{-k}+a_{0}^{*} a_{0} a_{k}^{*} a_{k}+a_{0}^{*} a_{0} a_{-k}^{*} a_{-k}=\left(a_{0}^{*} a_{k}+a_{-k}^{*} a_{0}\right)^{*}\left(a_{0}^{*} a_{k}+a_{-k}^{*} a_{0}\right)-a_{k}^{*} a_{k}-a_{0}^{*} a_{0},
$$

we deduce from (4.8) the estimate:

$$
\omega_{\Lambda, \rho}^{B}\left(a_{k}^{*}\left[H_{\Lambda}^{B}\left(\mu_{\Lambda}^{B}(\theta, \rho)\right), a_{k}\right]\right) \leq-\left[\varepsilon_{k}-\mu_{\Lambda}^{B}(\theta, \rho)-\frac{v(k)}{2 V}\right] \omega_{\Lambda, \rho}^{B}\left(N_{k}\right)+\frac{v(k)}{2 V} \omega_{\Lambda, \rho}^{B}\left(a_{0}^{*} a_{0}\right) .
$$

Therefore, combining (4.4) with (4.10) we find that:

$$
B_{k}\left(\mu_{\Lambda}^{B}(\theta, \rho)\right) \omega_{\Lambda, \rho}^{B}\left(N_{k}\right)-\beta \frac{v(k)}{2 V} \omega_{\Lambda, \rho}^{B}\left(a_{0}^{*} a_{0}\right) \leq \omega_{\Lambda, \rho}^{B}\left(N_{k}\right) \ln \frac{\omega_{\Lambda, \rho}^{B}\left(N_{k}\right)+1}{\omega_{\Lambda, \rho}^{B}\left(N_{k}\right)},
$$

with $B_{k}\left(\mu_{\Lambda}^{B}(\theta, \rho)\right)$ defined by (4.2). Notice that, since

$$
\mu_{\Lambda}^{B}(\theta, \rho)<\varepsilon_{\Lambda, 1}<\widehat{\varepsilon}_{\Lambda, 1}=\inf _{k \neq 0} \varepsilon_{k}
$$

and $\|k\|>2 \pi / L$, one has $B_{k}\left(\mu_{\Lambda}^{R}(\theta, \rho)\right)>0$. Hence we have to solve the inequality

$$
B_{k}\left(\mu_{\Lambda}^{B}(\theta, \rho)\right) x-\beta \frac{v(k)}{2 V} \omega_{\Lambda, \rho}^{B}\left(a_{0}^{*} a_{0}\right) \leq x \ln \frac{x+1}{x},
$$

for $x=\omega_{\Lambda, \rho}^{B}\left(N_{k}\right) \geq 0$. Notice that the solution of (4.12) is the set $\left\{0 \leq x \leq x_{2}\right\}$ where $x_{2}$ is a solution of the equation

$$
B_{k}\left(\mu_{\Lambda}^{B}(\theta, \rho)\right) x_{2}-\beta \frac{v(k)}{2 V} \omega_{\Lambda, \rho}^{B}\left(a_{0}^{*} a_{0}\right)=x_{2} \ln \frac{x_{2}+1}{x_{2}} .
$$

Let

[1] N. N. Bogoliubov, J. Phys.(USSR) 11, 23 (1947).

[2] N. N. Bogoliubov, Lectures on Quantum Statistics Vol. I:

$$
x_{1}=\frac{1}{e^{B_{k}\left(\mu_{\Lambda}^{B}(\theta, \rho)\right)}-1}
$$

be a nontrivial solution of the equation

$$
B_{k}\left(\mu_{\Lambda}^{B}(\theta, \rho)\right) x=x \ln \frac{x+1}{x}
$$

Then the inequality $x \leq x_{2}$ can be rewritten as

$$
x \leq x_{1}+\left(x_{2}-x_{1}\right)
$$

Since the function $f(x) \equiv x \ln \frac{x+1}{x}$ defined for $x \geq 0$ is concave, we get

$$
\frac{f\left(x_{2}\right)-f\left(x_{1}\right)}{f^{\prime}\left(x_{1}\right)} \leq x_{2}-x_{1}
$$

from which by (4.13), (4.14) we get (4.1) for $\|k\|>2 \pi / L$. 
[3] J. Ginibre, Commun. Math. Phys. 8, 26 (1968).

[4] J.-B. Bru, V. A. Zagrebnov, Phys. Lett. A 244, 371 (1998).

[5] J.-B. Bru, V. A. Zagrebnov, J. Phys. A 31, 9377 (1998).

[6] R. Griffiths, J. Math. Phys. 5, 1215 (1964).

[7] K. Hepp, E. H. Lieb, Phys. Rev. A 8, 2517 (1973).

[8] D. Ruelle, Statistical Mechanics: Rigorous Results (Benjamin, New-York, 1969).

[9] N. N. Bogoliubov (Jr), J. G. Brankov, V. A. Zagrebnov, A. M. Kurbatov, N. S. Tonchev, Russian Math. Surveys 39(6), 1 (1984).

[10] T. Michoel, A. Verbeure, J. Math. Phys. 40, 1268 (1999).

[11] J.-B. Bru, V. A. Zagrebnov, Physica A 268, 309 (1999).

[12] M. van den Berg, J. T. Lewis, Physica A 110, 550 (1982).

[13] M. van den Berg, J. Math. Phys. 23, 1159 (1982).

[14] M. van den Berg, J. T. Lewis, Commun. Math. Phys. 81,
475 (1981).

[15] J. V. Pulè, J. Math. Phys. 24, 138 (1983).

[16] M. van den Berg, J. Stat. Phys. 31, 623 (1983).

[17] J.-B. Bru, V. A. Zagrebnov, Phys. Lett. A 247, 37 (1998).

[18] Vl. V. Papoyan, V. A. Zagrebnov, Helv. Phys. Acta 63, 557 (1990).

[19] M. Fannes, A. Verbeure, Commun. Math. Phys. 55, 125 (1977).

[20] M. Fannes, A. Verbeure, Commun. Math. Phys. 57, 165 (1977).

[21] N. Angelescu, A. Verbeure, V. A. Zagrebnov, J. Phys. A 25, 3473 (1992).

[22] N. Angelescu, A. Verbeure, Physica A 216, 388 (1995).

[23] N. Angelescu, A. Verbeure, V. A. Zagrebnov, J. Phys. A 30, 4895 (1997).

\title{
СЛАБОНЕІДЕАЛЬНИЙ БОЗЕ-ГАЗ БОГОЛЮБОВА
}

\author{
В. А. Загрєбнов \\ Центр теоретичной фізики, \\ Університет Медітеране (Екс-Марсель II) \\ Люміні-Каз 907, Марсель, F-13288, Седекс 09, Франиіл \\ E-mail: zagrebnov@cpt.univ-mrs.fr
}

Показано, що конденсащія слабонеідеального бозе-газу Боголюбова може мати дві стадії. Якщо взаємодія є такою, що тиск слабонеідеального бозе-газу не збігається з тиском ідеального бозе-газу, то слабонеідеальний бозе-газ може виявляти два типи коденсацій: незєичну конденсацію в нульовій моді завдяки взаємодії (у першій стадії) і зөичну (у загальному типу I) бозе-айнштайнівську конденсацію в модах, суміжних із нульовою, завдяки насиченості густини частинок (у другій стадії). 